\title{
LOCALIZAÇÃO DE ESCOLAS DO ENSINO FUNDAMENTAL COM MODELOS CAPACITADO E NÃO-CAPACITADO: CASO DE VITÓRIA/ES
}

\author{
Fabrício Broseghini Barcelos * \\ Departamento de Engenharia Industrial - PUC-RIO \\ fbb@ind.puc-rio.br \\ Nélio Domingues Pizzolato \\ Departamento de Engenharia Industrial - PUC/RIO \\ Programa Pós-Graduação Engenharia Civil - UFF \\ Luiz Antonio Nogueira Lorena \\ Laboratório Computação e Matemática Aplicada - INPE \\ * Corresponding author/autor para quem as correspondências devem ser encaminhadas \\ Recebido em 06/2003; aceito em 03/2004 após 1 revisão \\ Received June 2003; accepted March 2004 after one revision
}

\section{Resumo}

O presente estudo está dividido em três partes. Na primeira parte, o objetivo do estudo é avaliar a atual localização das escolas públicas, identificando regiões onde há excesso ou escassez de vagas. Para a aplicação prática reportada, foram usados os setores censitários do IBGE, bem como suas informações de população escolarizável em cada setor, enquanto que para o cálculo dos diversos indicadores foi utilizado o software Arcview. Na segunda parte, apresenta-se uma proposta de relocalização resultante da aplicação do modelo da p-mediana. Essa proposta sugere uma distribuição ideal que leva em conta os rearranjos demográficos da área estudada. A terceira parte do estudo considera o modelo da p-mediana capacitado e refaz o estudo de relocalização, mas supondo unidades escolares com capacidade definida. $\mathrm{O}$ estudo foi conduzido com as escolas públicas do ensino fundamental na cidade de Vitória/ES, com cerca de 300.000 habitantes, 271 setores censitários e 45.766 escolares na idade 7-14 anos.

Palavras-chave: localização de escolas; modelo das p-medianas; sistemas de informação geográfica.

\begin{abstract}
The present study is divided in three parts. In the first part, the objective of the study is to evaluate the current location of public schools. The spatial distribution is evaluated with the p-median model and the Arcview, a GIS software. Given the existing capacity, regions with excess or shortage of school places have been identified. In the second part, a heuristic algorithm to solve the p-median problem has been used and the proposed locations identified. This study proposes an ideal distribution of the school network which takes into consideration the recent demographic modifications experienced in the area. The third part of the study assumes that schools have a single standard size and considers the capacitated p-median using an special purpose algorithm. The study was applied to the location of elementary public schools in the city of Vitória, a state capital with about 300,000 inhabitants located in the southeast part of Brazil. The number of school children is 45,766 with 51 public schools, according to the 2000 National Census.
\end{abstract}

Keywords: school location; p-median model; geographic information systems. 


\section{Introdução}

Este trabalho tem como objetivo estudar a problemática da localização de escolas públicas de ensino fundamental em grandes centros urbanos. Para tanto, o problema foi examinado sob três óticas distintas, a saber: (i) um procedimento de avaliação de escolas públicas com respeito à atual localização e atual capacidade, baseando-se na situação existente, (ii) uma proposta de localização de escolas, na qual procura-se apontar como as escolas deveriam se distribuir espacialmente e (iii) o mesmo problema anterior, mas supondo que cada escola seja uma unidade com capacidade pré-estabelecida.

Os dois primeiros estudos supõem modelos não capacitados, enquanto o terceiro corresponde ao problema capacitado. Entretanto, como toda unidade escolar possui limites de capacidade efetiva, nos dois primeiros estudos esta capacidade é introduzida, não no modelo em si, mas na avaliação gerencial dos resultados práticos do modelo. $\mathrm{O}$ terceiro estudo busca superar essa limitação. Para todos esses estudos usou-se o princípio que norteia o uso do modelo da p-mediana, a saber: todo aluno deve freqüentar a escola mais próxima de sua residência.

O estudo tomou como base o município de Vitória, capital do estado do Espírito Santo com área de 88,8 quilômetros quadrados, população de 291.941 habitantes e uma das maiores densidades demográficas do país com 3.288,90 habitantes por quilômetro quadrado. A metodologia aplicada supõe que toda a população na faixa etária de 7-14 anos deve estar matriculada no ensino fundamental, seja numa escola municipal, estadual ou federal.

Na sequiência, para ressaltar os aspectos práticos do estudo, tanto a teoria como sua aplicação à cidade de Vitória são apresentadas de modo integrado. Assim, a Seção 2 examina o uso de sistemas de informação geográficos, a Seção 3 apresenta a descrição metodológica referente ao estudo de avaliação do estado atual, as Seções 4 e 5 ressaltam a apresentação dos elementos gerenciais do primeiro estudo, a Seção 6 executa o segundo estudo, ou seja, a proposta de localização, a Seção 7 desenvolve o terceiro estudo, em que as escolas são capacitadas, em tamanhos padronizados, a Seção 8 faz uma síntese dos resultados, enquanto que a Seção 9 enumera as conclusões da pesquisa.

\section{Avaliação da Atual Localização com Sistemas Geográficos}

Em todo o estudo foi extensamente usado o software Arcview, da família dos Sistemas de Informação Geográficos, SIG. Esses sistemas de informação geográficos são ferramentas que associam bancos de dados com informações espaciais, na forma de mapas digitalizados, identificando mapas associados a dados relativos à geografia da região. Além de favorecer a representação visual das análises, esses sistemas permitem efetuar diversas operações entre banco de dados descritivos de ambientes geográficos. Em particular, aproveita-se a vantagem do software adotado para:

- Calcular a área de abrangência de cada escola;

- Servir como Banco de Dados;

- Simplificar a tarefa de manipulação de dados;

- Melhor compreensão e apresentação do problema;

- Melhor análise dos resultados obtidos por meio da visualização espacial. 
Na parte aplicada do presente estudo foram adquiridos dados junto ao Instituto Brasileiro de Geografia e Estatísticas (IBGE), que vem, de modo sistemático, digitalizando os mapas dos principais centros urbanos brasileiros, assim como disponibilizando dados censitários diversos, inclusive os específicos da população escolarizável.

Os sistemas geográficos, basicamente, permitem organizar as informações sobre uma determinada região em camadas, onde cada camada, que representa um tema ou um layer, é um conjunto de feições homogêneas que exibem uma informação a respeito de uma característica da região e estão posicionalmente relacionadas às outras camadas por meio de um sistema de coordenadas comuns. As informações exibidas nas diferentes camadas podem ser analisadas separadamente ou em combinação com outras, uma vez que nem todas as análises necessitam do uso de todos os layers simultaneamente. Localizações ou áreas de um determinado tema podem também ser extraídos dos mesmos, separando-os de localizações vizinhas e criando novos layers.

Além disso, os sistemas geográficos em geral, e o Arcview em particular, permitem ainda junções espaciais ligando colunas de tabelas distintas a partir da localização geográfica das entidades. $\mathrm{Na}$ junção de duas tabelas, dependendo do tipo de entidade (ponto, linha ou polígono) que compõe cada tema, o programa procura qual entidade se encontra dentro ou mais próximo de outra entidade. Dessa forma, encontram-se linhas, pontos ou polígonos que estejam inseridos em outros polígonos, além de calcular a menor distância entre pontos de tabelas diferentes.

\section{Descrição Metodológica para Obtenção da Área de Atuação de Cada Escola}

O primeiro passo a ser dado para estudar a área de atuação de cada escola é procurar discretizar a distribuição da população, evitando o modo contínuo, que impossibilita estudos normativos. Dado o mapa da região a ser estudada, ela deverá estar dividida em setores censitários, que são pequenas divisões definidas pelo IBGE, a fim de facilitar a realização do Censo, pois um único recenseador é o responsável pelo levantamento de dados nesta área num limitado espaço de tempo. No estudo de caso abordado, relativo à cidade de Vitória, existem 271 setores censitários, como mostrado nos polígonos da Figura 1.

Para cada setor censitário é preciso localizar seu respectivo centróide, ou seja, um ponto representativo do centro demográfico do setor. Como não há como conhecer o exato posicionamento de cada aluno, assume-se que toda a população se encontra localizada no referido centróide. Em áreas urbanas, essa suposição é correntemente adotada e, tratando-se de áreas pequenas, não parece provocar distorções significativas.

O próximo passo consiste em obter os dados referentes ao último recenseamento (Censo 2000) no que tange à população em faixa escolar, em particular, as quantidades de crianças na faixa etária de 7-14 anos em cada setor censitário. Na falta de acesso aos dados de faixa etária por setor censitário, foi adotada a seguinte simplificação: Como o referido Censo apontou uma população total de 292.304 habitantes, e a correspondente pirâmide etária indicou uma população de 45.766 jovens na faixa 7-14 anos, segue-se que $15,6 \%$ da população estava na idade escolarizável do ensino fundamental. A partir daí, supôs-se que essa proporção seria uniforme para todos os setores censitários.

A seguir, na Secretaria Estadual de Educação (SEDU/ES), foram obtidos os endereços das escolas públicas de ensino fundamental existentes e o número de matriculados por escola. 
A Figura 1 também mostra a distribuição espacial e localização de todas as escolas estaduais e municipais de Vitória/ES, mediante pontos no mapa.

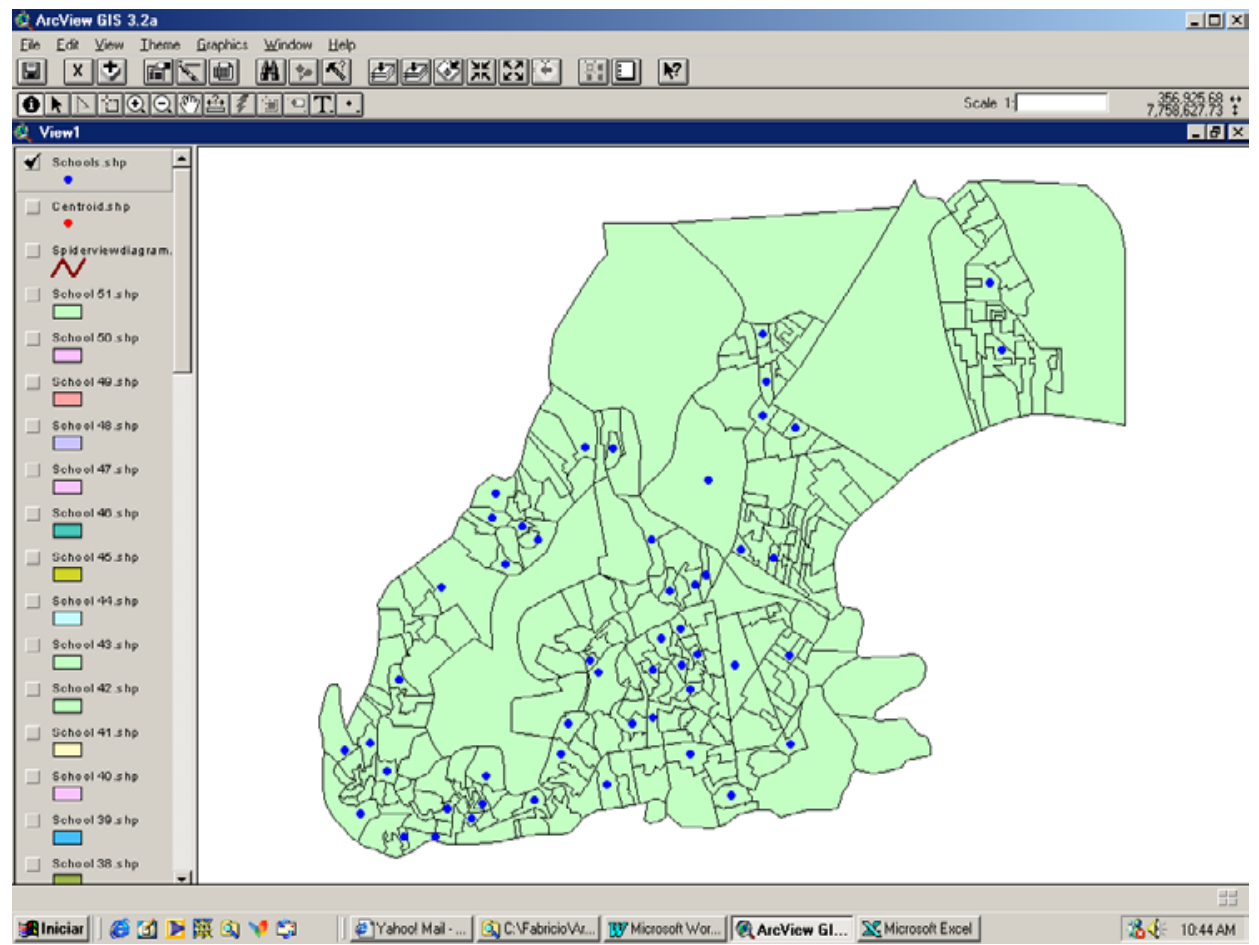

Figura 1 - Setores Censitários e Distribuição Espacial das Escolas Públicas de Vitória

O modelo das p-medianas para localizar escolas, ou avaliar sua atual distribuição geográfica, tem como base as seguintes premissas:

- Toda a população em idade escolar que mora num determinado setor censitário encontrase concentrada no seu respectivo centro demográfico (centróide);

- Todas as escolas oferecem as mesmas condições de ensino, conservação, espaço para recreação, conforto, merenda escolar, tradição, qualificação dos professores, acesso a séries ou níveis de ensino posteriores etc. Dessa forma, não se justifica nenhum aluno evitar a escola mais próxima de sua residência, podendo, assim, se deslocar a pé até a mesma. Em outras palavras, se a escola mais próxima da residência oferece condições idênticas ou bem parecidas à escola modelo, então o critério de proximidade passa a ser um determinante na seleção desta pelo usuário. Essa hipótese simplificadora foi empiricamente comprovada em Pizzolato \& Silva (1993) ao estudar o comportamento dos alunos na área de Nova Iguaçu/RJ;

- Escolas localizadas num mesmo setor censitário atendem à mesma área e são consideradas apenas uma, com as capacidades somadas. Em Vitória existem 54 escolas públicas, porém, para efeito deste estudo, foram consideradas 51 escolas, pois três setores censitários apresentam duas escolas cada um. 
Como o objetivo do trabalho, de acordo com a segunda premissa acima, é minimizar a distância média percorrida escola-centróide, deve-se, a seguir, determinar qual setor censitário se encontra mais próximo de cada escola. Em outras palavras, determina-se a área de abrangência de cada escola. Para tanto, foi utilizada a função Junção entre Tabelas do Arcview, que une duas tabelas de layers compostos de pontos e calcula a menor distância entre estes pontos.

A seguir, seleciona-se os setores censitários mais próximos de cada escola separadamente e cria-se um novo layer para cada área de abrangência.

\section{Descrição Metodológica para Obtenção do Excesso ou Escassez de Vagas em Cada Escola}

Para obter-se o excesso ou escassez de vagas no ensino fundamental de Vitória, foi feita uma análise de cada escola separadamente, com sua respectiva área de abrangência. De posse da tabela dos centróides, pode-se calcular a demanda somando-se individualmente a coluna Demanda Centróide para cada escola. Por outro lado, o Arcview facilita este trabalho através da função Summarize, criando automaticamente uma nova tabela com estes somatórios.

Primeiramente deve-se selecionar na tabela dos centróides a coluna IdEscola. No menu Field, escolhe-se a opção Summarize. Na janela Summary Table Definition, clica-se na opção Save As para a escolha do diretório onde se deseja salvar o arquivo suml.dbf, criado automaticamente pelo Arcview com a opção Summarize. A seguir, nos campos Field e Summarize by escolhe-se as opções Demanda Centróide e Sum, respectivamente. Clique em Add e em seguida $O k$.

Dessa forma, cria-se uma nova tabela chamada suml.dbf com dois novos campos: Count e Sum_Demanda Centróide. O campo count mostra o número de setores censitários atraídos por cada escola, enquanto que o campo Sum_Demanda Centróide mostra a demanda de cada escola.

Deste modo, pode-se verificar em cada área de abrangência o número de crianças em idade escolar. Em outras palavras, a demanda de cada escola é definida como sendo o número de crianças morando em todos os setores contidos em cada árvore.

Por último, é preciso comparar os dados referentes ao número de crianças de cada área de abrangência com as respectivas capacidades de cada escola (dados fornecidos pela Secretaria de Educação do Estado). Para tanto, criam-se duas novas colunas na tabela suml.dbf: a primeira chamada Capacidade e a segunda chamada Excesso/Escassez.

Após digitar as respectivas capacidades das escolas, pode-se determinar o excesso e a escassez de vagas para cada segmento da escola. A coluna Excesso/Escassez é preenchida subtraindo da coluna Capacidade a coluna Sum_Demanda Centróide. Se o resultado na coluna Excesso/Escassez for negativo, significa que a escola está com escassez de vagas. Caso contrário, se o valor der positivo, significa que a escola apresenta excesso de vagas e que recursos podem ser remanejados para áreas mais necessitadas.

Assim sendo, nas regiões onde se nota um esvaziamento populacional ou uma redução na taxa de natalidade, apresenta-se um certo excesso de vagas, o estudo pode apontar quais as escolas que podem ser fechadas ou reduzidas em suas capacidades. Por outro lado, pode-se 
avaliar possíveis expansões da rede escolar nas regiões que apresentam crescimento demográfico, como é o caso de algumas regiões em Vitória. Esta expansão pode ser feita por meio da construção de novas escolas ou pela ampliação de escolas já existentes.

\section{Avaliação da Atual Localização}

Nesta etapa, é necessário conhecer a localização das 51 escolas públicas existentes, cada uma associada a um determinado setor censitário e indicadas por meio de arquivo do Bloco de Notas (.txt). O grafo é então dividido em 51 árvores que correspondem às 51 micro regiões, indicando o espaço de abrangência de cada escola, de acordo com a proximidade, de modo que cada setor passa a integrar a micro região de sua escola mais próxima. Nessa etapa, não há nenhuma tentativa de otimização, simplesmente de constatação. Trata-se de um procedimento elementar de comparar, para cada setor, sua distância a todas as escolas existentes, e identificar a mais próxima. Essa operação pode ser realizada pelo próprio ArcView e também por um algoritmo elementar que facilite a posterior emissão de relatório de excesso ou de escassez de vagas naquela micro região definida por cada escola e que atende a todos os setores censitários mais próximos.

No caso, ambos os procedimentos foram adotados e, como resultado, o algoritmo elementar fornece um relatório contendo, primeiramente, o valor da função objetivo " $Z$ ” encontrado para o município. A seguir, para cada uma das árvores criadas, o algoritmo fornece o código da mediana escolhida, a quantidade de vértices atraídos por esta mediana e os códigos dos vértices atraídos. Além disso, o algoritmo fornece a contribuição "J" de cada árvore no valor "Z" da função objetivo, a maior distância " $D$ " percorrida dentro de cada árvore e qual o vértice mais distante.

Para exemplificar o relatório obtido, tome-se como exemplo a árvore cuja raiz encontra-se no vértice 221, associada a uma escola, sobre a qual tem-se as informações:

- Vértice mediano: Vértice 221;

- Número de vértices atraídos: 14;

- Vértices atraídos: 221, 3, 4, 5, 6, 7, 8, 9, 10, 11, 12, 21, 22, 23;

- Contribuição na função objetivo: $\mathrm{J}(221)=6559$;

- Maior distância percorrida dentro da árvore: $\mathrm{D}(221)=1503$;

- Vértice mais distante da mediana escolhida: Vértice $12(\mathrm{~J}=12)$.

A Figura 2 é uma tentativa de visualizar o problema existente. Essa figura pode receber cores, de acordo com um código que indique a gravidade dos desequilíbrios encontrados, segundo faixas de excesso ou escassez de vagas nas micro-regiões encontradas. Esse estudo é importante para o gerenciamento de curto prazo do conjunto da rede escolar. Dessa forma, fica claro observar que as áreas prioritárias de investimentos deveriam ser aquelas com coloração destacada, pois representam áreas com escassez superior a 1000 vagas. 


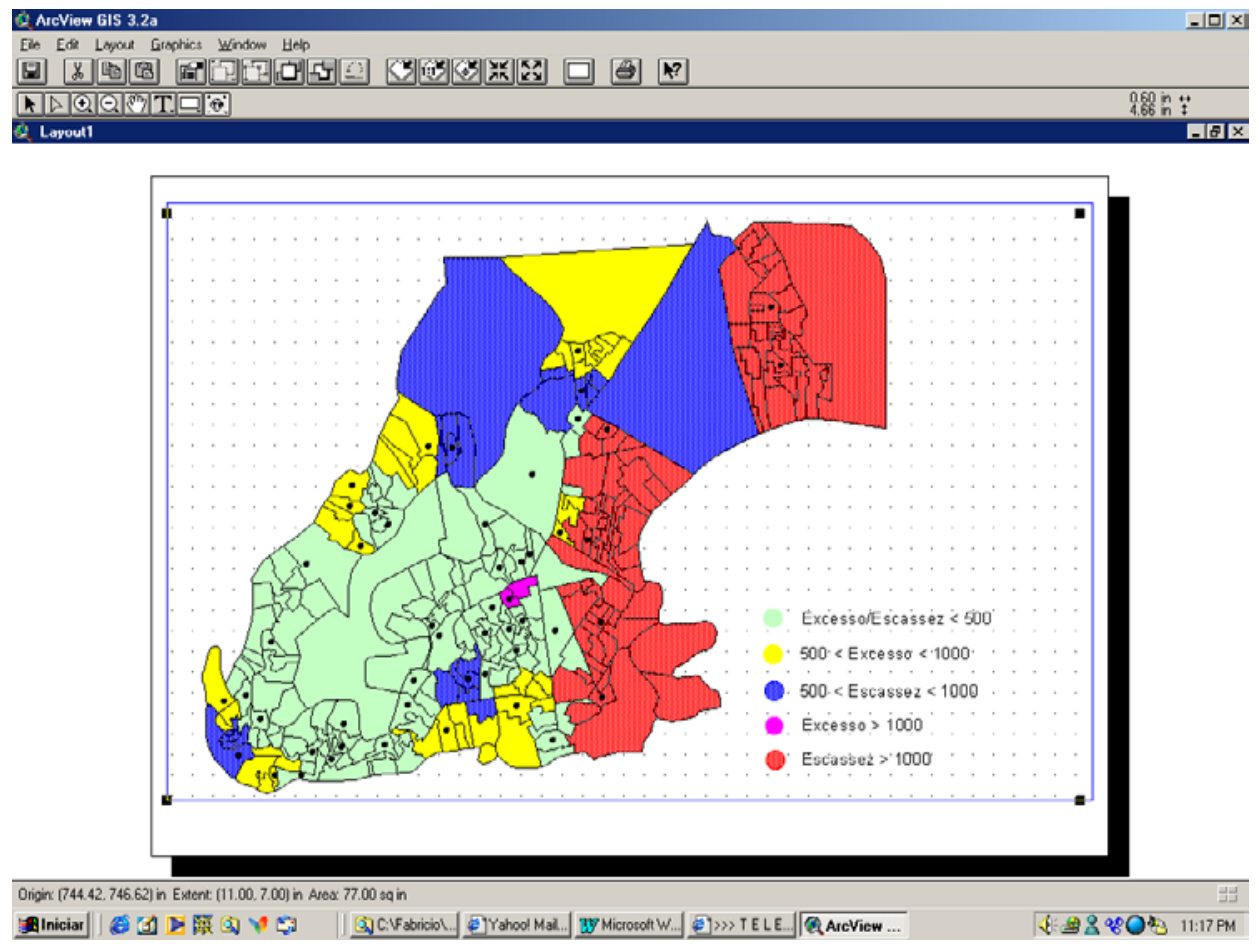

Figura 2 - Avaliação da Atual Situação por Faixas de Excesso ou Escassez de Vagas

\section{Proposta de uma Nova Localização}

Para estudar a distribuição espacial e fazer uma proposta de localização, o problema foi modelado como a p-mediana. Sua solução pode ser obtida por métodos heurísticos e métodos com base em programação matemática. Aproximações heurísticas são mais populares por se tratar geralmente de natureza mais simples, são convenientes em problemas de grande escala e podem oferecer boas soluções. Métodos exatos, como Galvão \& Raggi (1989), requerem a solução do problema de programação inteira, associada a diversos recursos de otimização. Dentre os métodos heurísticos desenvolvidos para o problema das p-medianas, o de Pizzolato (1994), aplicado neste trabalho, parece ser adequado no caso de grandes redes.

Nesta etapa, foi aplicado o referido algoritmo para determinar a localização ideal de 51 escolas. Com a resposta obtida, o grafo é então dividido em 51 árvores, que correspondem a 51 micro-regiões, que podem vir a ser significativamente distintas daquelas notadas na primeira etapa do estudo.

O resultado da proposta tende a criar micro regiões mais uniformes e balanceadas ao longo do município. Importante notar, no quadrilátero nordeste do município, que o modelo propõe a localização de cinco escolas, em contraposição às duas existentes nas Figuras 1 e 2. Tratase de uma região em processo de urbanização muito rápida onde o sistema público não vem acompanhando o desenvolvimento urbano.

Novamente, como resposta o algoritmo fornece um relatório contendo, primeiramente, o valor da função objetivo " $Z$ ” encontrado. A seguir, para cada uma das árvores criadas, o 
algoritmo fornece o código da mediana escolhida, a quantidade de vértices atraídos por esta mediana e os códigos destes vértices. Além disso, o algoritmo fornece a contribuição "J" de cada árvore no valor " $Z$ " da função objetivo, a maior distância " $D$ " percorrida dentro de cada árvore e qual o vértice mais distante. Tomando-se, como exemplo, a árvore associada ao vértice 174 , tem-se:

- Vértice escolhido como mediana: Vértice 174;

- Número de vértices atraídos: 06;

- Vértices atraídos: 168, 173, 174, 176, 177 e 265;

- Contribuição na função objetivo: $\mathrm{J}(174)=3557$;

- Maior distância percorrida dentro da árvore: $\mathrm{D}(174)=518$;

- Vértice mais distante da mediana escolhida: Vértice 168 ( $\mathrm{J}=168)$.

A conciliação da proposta de localização com o fato de existirem escolas operando no município pode ser feita com um raciocínio simples. Assim, as localizações propostas dividem a área em 51 micro regiões, e cada escola existente pertencerá a uma delas, agregando para ela a sua capacidade. Dessa forma, embora as atuais escolas possam não coincidir com as localizações propostas, elas oferecerão capacidade de atendimento às micro regiões apontadas pelo modelo. Assim, para cada micro região proposta haverá uma demanda de seus estudantes e oferta pela capacidade das escolas existentes. Os resultados dessa avaliação comparativa estão presentes na Figura 3.

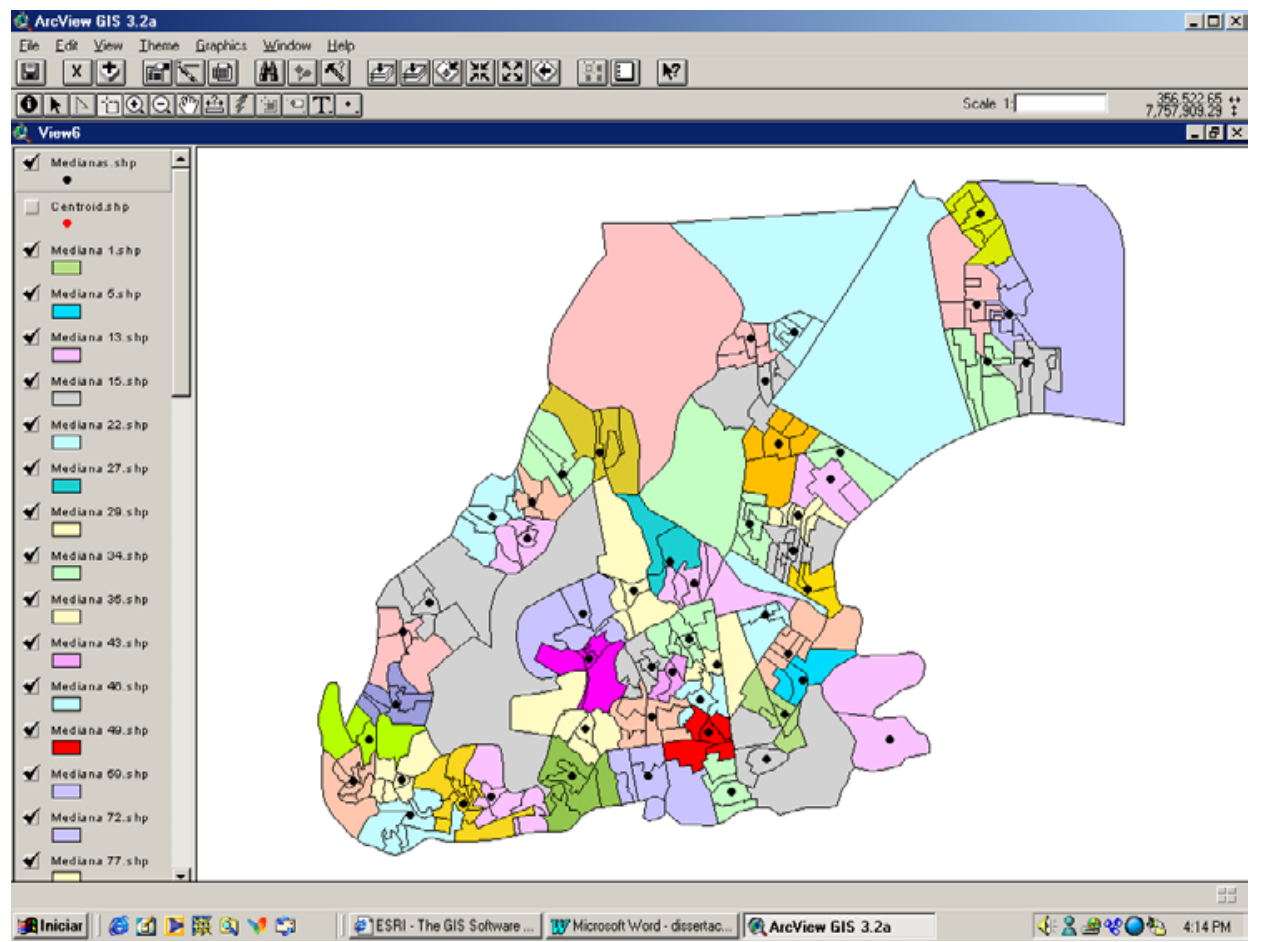

Figura 3 - Solução do Modelo Não-Capacitado 


\section{Heurística Lagrangeana/Surrogate (Modelo Capacitado)}

O problema das p-medianas capacitado (PMC) considera a capacidade a ser dada por cada mediana, ou seja, a oferta de vagas em cada região. Parte da premissa que a demanda total dos vértices alocados a uma mediana não pode ser maior que a capacidade da respectiva escola.

Esta aplicação pode ser importante nos casos em que a administração deseja construir escolas padronizadas, ou seja, com a mesma capacidade. Estudos anteriores mostram que o custo por estudante é maior tanto em escolas muito pequenas, quanto em escolas muito grandes, fazendo sentido a busca de escolas padronizadas em tamanhos considerados médios.

A solução do problema é obtida através da Heurística Lagrangeana/Surrogate ou "Lagsur" proposta em Narciso \& Lorena (1999). A relaxação Lagrangeana/Surrogate desenvolvida para resolver de forma aproximada o problema das p-medianas com restrições de capacidade apresenta melhores resultados que a relaxação Lagrangeana usual, obtendo limitantes de igual qualidade com menor esforço computacional

Segundo Lorena et al. (2001), a formulação do problema capacitado é a seguinte:

$$
\begin{array}{ll}
(P M C)=\operatorname{Min} \quad \sum_{i=1}^{n} & \sum_{j=1}^{n} d_{i j} x_{i j} \\
\text { sujeito } a & \sum_{j=1}^{n} x_{i j}=1 ; i \in N \\
& \sum_{j=1}^{n} x_{j j}=p \\
& \sum_{i \in N} q_{i} x_{i j} \leq Q_{j} x_{j j} ; \mathrm{j} \in \mathrm{N} \\
& x_{i j} \in\{0,1\} ; i, j \in N
\end{array}
$$

onde,

$\mathrm{q}_{\mathrm{i}}=$ demanda associada ao vértice $\mathrm{i}$

$\mathrm{Q}_{\mathrm{j}}=$ capacidade da escola $\mathrm{j}$

$\left[\mathrm{x}_{\mathrm{ij}}\right]_{\mathrm{n} \times \mathrm{n}}$ é uma matriz de alocação, com $\mathrm{x}_{\mathrm{ij}}=1$ se o setor (vértice) $\mathrm{j}$ é atendido pela escola (mediana) $\mathrm{i}$, e $\mathrm{x}_{\mathrm{ij}}=0$, caso contrário

$\left[d_{i j}\right]_{n x m}$ é uma matriz de distâncias

p é o número de medianas;

n é o número de nós, e $\mathrm{N}=\{1, \ldots, \mathrm{n}\}$.

As restrições (2) e (3) garantem que cada nó é alocado a somente uma mediana. A restrição (4) impõe que a capacidade total das medianas deve ser respeitada, e (5) define as variáveis binárias.

Inicialmente uma relaxação surrogate é aplicada ao $P M C$ gerando uma restrição que substituirá as restrições (2). Em seguida é aplicada uma relaxação Lagrangeana da restrição surrogate, gerando a relaxação conhecida como Lagrangeana/surrogate. 
Considere o multiplicador $\lambda \in R^{n}$ a restrição surrogate relativa à restrição (2) será:

$$
\sum_{i \in N} \sum_{j \in N} \lambda_{i} x_{i j}=\sum_{i \in N} \lambda_{i}
$$

e para um multiplicador $t \in \mathrm{R}$, a Relaxação Lagrangeana/Surrogate de $P M C$ será:

$$
v\left(L_{t} P M C^{\lambda}\right)=\operatorname{Min} \sum_{i \in N} \sum_{j \in N}\left(d_{i j}-t \lambda_{i}\right) x_{i j}+t \sum_{i \in N} \lambda_{i}
$$

sujeito a

(3), (4) e (5).

O problema $\left(L_{t} P M C^{\lambda}\right)$ é solucionado decompondo-se em n problemas da mochila 0-1:

$$
v\left(\text { knap }_{j}\right)=\operatorname{Min} \sum_{i \in N}\left(d_{i j}-t \lambda_{i}\right) x_{i j} ; \mathrm{j} \in \mathrm{N}
$$

sujeito a (4) e (5).

Cada um destes problemas da mochila é resolvido usando o algoritmo de Horowitz e Sahni (descrito em Martello \& Toth, 1990). Seja I o conjunto de índices dos p menores $v\left(k_{n a p}\right)$, $\mathrm{j} \in \mathrm{N}$ (neste ponto a restrição (3) está sendo tratada implicitamente). $\mathrm{O}$ valor Lagrangeano/surrogate é dado por:

$$
\mathrm{v}\left(\mathrm{L}_{\mathrm{t}} \mathrm{PMC}^{\lambda}\right)=\sum_{j \in I} v\left(k_{n a p}\right)+t \sum_{i \in N} \lambda_{i}
$$

Uma característica interessante da relaxação $\left(L_{t} P M C^{\lambda}\right)$ é que, para $t=1$ tem-se a relaxação Lagrangeana usual considerando o multiplicador $\lambda$, e para um multiplicador $\lambda$ fixo, o melhor valor para $t$ pode ser encontrado resolvendo-se o problema dual Lagrangeano

$$
\mathrm{v}\left(\mathrm{D}_{\mathrm{t}}^{\lambda}\right)=\underset{t \geq 0}{\operatorname{Max}} v\left(L_{t} P M C^{\lambda}\right) \text {. }
$$

Sabe-se que $\mathrm{v}\left(\mathrm{L}_{\mathrm{t}} \mathrm{PMC}^{\lambda}\right)$ como função de t, é côncava e linear por partes. Assim, em geral, existe um intervalo de valores $\mathrm{t}_{0} \leq \mathrm{t} \leq \mathrm{t}_{1}\left(\operatorname{com~t}_{0}=1\right.$ ou $\left.\mathrm{t}_{1}=1\right)$ no qual tem-se $\mathrm{v}\left(\mathrm{L}_{\mathrm{t}} \mathrm{PMC}^{\lambda}\right)>$ $\mathrm{v}\left(\mathrm{L}_{1} \mathrm{PMC}^{\lambda}\right)$, como ilustra a Figura 4 a seguir (para o caso de $\mathrm{t}_{1}=1$ ).

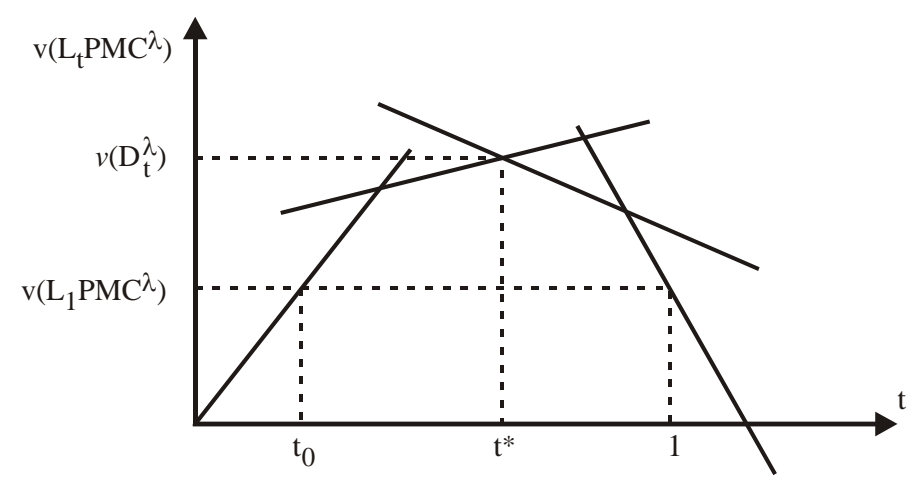

Figura 4 - Limitantes na função $\mathrm{L}_{\mathrm{t}} \mathrm{PMC}^{\lambda}$ 
Assim, para obter um limitante melhor do que o obtido pela relaxação Lagrangeana usual não é necessário determinar o melhor valor $t^{*}$, sendo suficiente encontrar um valor $\mathrm{T}$ tal que $\mathrm{t}_{0}<\mathrm{T}<\mathrm{t}_{1}$ (sendo $\mathrm{t}_{1}=1$ na Figura 4 ). Este valor foi encontrado através de um procedimento de busca heurística descrito em Senne \& Lorena (2000).

O seguinte algoritmo de subgradientes é utilizado para resolver o problema de p-medianas.

Dados $\lambda \geq 0, \lambda \neq 0$, fazer $\mathrm{lb}=-\infty, \mathrm{ub}=+\infty$;

Repetir

Resolver a relaxação $\left(\mathrm{L}_{\mathrm{t}} \mathrm{PMC}^{\lambda}\right)$ obtendo $\mathrm{x}^{\lambda}$ e $\mathrm{v}\left(\mathrm{L}_{\mathrm{t}} \mathrm{PMC}^{\lambda}\right)$;

Obter uma solução viável $\mathrm{x}^{\mathrm{f}}$ e atualizar o valor $\mathrm{v}^{\mathrm{f}}$ correspondente (resolvendo o problema PGA definido abaixo);

Atualizar o limite inferior $\mathrm{lb}=\operatorname{Max}\left[\mathrm{lb}, \mathrm{v}\left(\mathrm{L}_{\mathrm{t}} \mathrm{PMC}^{\lambda}\right)\right]$;

Atualizar o limite superior $\mathrm{ub}=\operatorname{Min}\left[\mathrm{ub}, \mathrm{v}^{\mathrm{f}}\right]$;

Fazer $g_{i}^{\lambda}=1-\sum x_{i j}^{\lambda}, i \in N$;

Atualizar o tamanho do passo $\theta$;

Fazer $\lambda_{i}=\lambda_{i}+\theta g_{i}^{\lambda}, i \in N$;

Enquanto (as condições de parada não forem verificadas).

O valor inicial de $\lambda$ é $\lambda_{i}=\min _{j \in N}\left\{d_{i j}\right\}, i \in \mathrm{N}$, o tamanho do passo é calculado como: $\theta=\left[\pi(u b-l b) /\left\|\mathrm{g}^{\lambda}\right\|^{2}\right]$, e as condições de parada foram: número de iterações maior do que 1000 , ou $\pi \leq 0.005$, ou $\mathrm{ub}-\mathrm{lb}<1$, ou $\left\|\mathrm{g}^{\lambda}\right\|^{2}=0$ (solução ótima).

O controle do parâmetro $\pi$ é o proposto por Held \& Karp (1971), onde $0 \leq \pi \leq 2$. Inicialmente seu valor é fixado em $\pi=2$, sendo reduzido à metade em cada iteração sempre que $l b$ mantiver seu valor constante por 30 iterações sucessivas.

A solução $x^{\lambda}$ do $v\left(L_{t} P M C^{\lambda}\right)$ não é necessariamente viável, mas o conjunto $I$ identifica os vértices escolhidos como centros que podem ser usados para produzir soluções viáveis para os problemas. O problema PMC com medianas fixadas é reduzido a outro problema de otimização combinatória, o problema generalizado de atribuição, onde itens (vértices) são atribuídos a mochilas (medianas) respeitando suas respectivas capacidades e maximizando o retorno obtido com essas atribuições. Para alocar os vértices não-medianas no conjunto de medianas identificado anteriormente, deve-se solucionar o seguinte problema generalizado de atribuição:

(PGA)

$$
\begin{array}{ll}
\operatorname{Max} & \sum_{i \in N-I} \sum_{j \in I} p_{i j} x_{i j}^{f} \\
\text { sujeito a: } & \sum_{i \in N-I} q_{i} x_{i j}^{f} \leq Q_{j}, \mathrm{j} \in \mathrm{I}
\end{array}
$$




$$
\begin{aligned}
& \sum_{j \in I} x_{i j}^{f}=1, \mathrm{i} \in \mathrm{N}-\mathrm{I} \\
& x_{i j}^{f} \in\{0,1\}, \mathrm{i} \in \mathrm{N}-\mathrm{I} ; \mathrm{j} \in \mathrm{I}
\end{aligned}
$$

Sendo $p_{i j}=-d_{i j}, i \in \mathrm{N}-I ; j \in I$, o ganho se o vértice $i$ for atribuído ao centro $j$.

$x_{i j}^{f}=1$ se o vértice $i$ é alocado ao centro $j \in C$ e $x_{i j}^{f}=0$, caso contrário.

O algoritmo $M T H G$ proposto por Martello \& Toth (1990) é usado para resolver de forma aproximada o problema generalizado de alocação (PGA). A solução $x^{f}$ do problema acima é ainda melhorada por uma Heurística de Localização-Alocação apresentada em Lorena \& Senne (2003).

Essa heurística baseia-se na observação que, após a definição de $x^{f}$, obtém-se exatamente $p$ aglomerados identificados por $\mathrm{C}_{1}, \mathrm{C}_{2}, \ldots, \mathrm{C}_{\mathrm{p}}$, correspondendo às $\mathrm{p}$ medianas e suas respectivas não-medianas alocadas. A solução $x^{f}$ pode ser melhorada procurando-se por um novo centro dentro de cada aglomerado, trocando-se a mediana atual por outro vértice e recalculando-se as alocações.

O algoritmo de p-medianas descrito anteriormente foi integrado por Lorena \& Senne (2003) a dois Sistemas de Informações Geográficas: Arcview (ESRI, 1996) e SPRING (SPRING, 1998). Para efeito deste estudo, considerando a base de dados reais da cidade de Vitória, foi testada somente a integração com o Arcview.

A distância entre os vértices foi calculada a partir da escala do mapa no qual estão inseridos. Os valores resultantes representam a distância direta linear entre os vértices ou a distância sobre os arcos que compõem o mapa. Neste modelo de solução do problema de p-medianas, a distância entre os vértices foi o único parâmetro de custo considerado.

Para a visualização da solução, utilizou-se a função Spider, disponível no Arcview, que foi modificada para se adequar às necessidades da integração. Essa função verifica as distâncias entre os vértices de demanda, contidos em um tema, e os vértices relativos aos centros ofertantes, contidos em outro tema, e representa a alocação dos vértices aos centros selecionados para atendimento.

Os valores de demanda podem ser extraídos dos temas disponíveis baseados no número de imóveis existentes em cada setor. Porém, os valores considerados neste trabalho, assim como na Seção 3, foram extraídos baseando-se no Censo Populacional elaborado pelo IBGE.

A partir dessa informação, a script calcula a demanda total como sendo a soma da demanda de todos os vértices do tema de pontos selecionado. Este valor é então dividido pelo número de medianas a serem localizadas, definindo, assim, a capacidade de cada centro de atendimento. $\mathrm{O}$ valor encontrado pode, ainda, ser multiplicado por um fator maior que 1, permitindo modelar cenários com escassez ou excesso na capacidade de atendimento dos centros.

Na Figura 5 tem-se a visualização da Spider com a solução do modelo capacitado para a região de Vitória considerando distâncias lineares. A Figura 6 destaca a necessidade de novas escolas na região norte do município, comparando a situação atual (duas escolas) e a proposta do modelo capacitado, que propõe cinco escolas. 


\section{Síntese dos Resultados}

Analisando a Figura 2 pode-se observar que há escassez de vagas em grande parte das regiões. Nos lugares onde há excesso de vagas, esse número não é suficiente para atender à falta de vagas das outras regiões. O somatório da demanda é de 45.766, enquanto que o somatório da oferta de vagas é de 42.198. Assim sendo, há uma falta global estimada na região de 3.568 vagas. Essa falta de vagas é decorrente de um cálculo aritmético simples que considera a capacidade total e a população apontada pelo IBGE. Entretanto, há quatro fatores que afetam seu cálculo, a saber: (i) a participação da escola privada, que absorve parte da demanda; (ii) a repetência, que mantém na escola alunos em faixas etárias superiores a 7-14 anos; (iii) a evasão escolar e (iv) a atração de alunos de municípios vizinhos.

Com relação à avaliação da atual situação, pode-se concluir que:

- O estudo mostra onde a construção de mais escolas é necessária. Algumas regiões do município, como por exemplo a zona norte, são mal atendidas pelo sistema público. Isto pode ser explicado pelo fato dessas regiões serem habitadas por pessoas de renda mais elevada, que tradicionalmente preferem escolas privadas;

- A falta de um planejamento acompanhando o processo de urbanização da cidade fez com que os órgãos competentes não se alertassem para a explosão demográfica de certas regiões, tal como a região do município destacada na Figura 5, região que conta com apenas 2 escolas, como ressaltado na área focalizada. Na Figura 6 estão tanto a solução atual, como a solução capacitada. Tanto essa última, como a proposta de relocalização, recomendam 5 escolas na área;

- O gerenciamento da capacidade a curto prazo, foco de interesse na primeira parte do estudo, pode ser facilmente atendido com medidas simples, tais como ativando espaços não usados, contratando mais professores, adaptando salas existentes porém vazias etc.

Com relação à proposta de uma nova localização para as escolas obtida pelo Algoritmo de Pizzolato, podemos concluir que:

- Fechamento ou redução na capacidade das escolas localizadas na região central de Vitória. A região central, antes densamente povoada, vem sofrendo uma séria migração nas últimas décadas. Dessa forma, as escolas que foram planejadas para uma demanda muito maior que a atual, estão com excesso de vagas;

- A natureza não-capacitada do modelo empregado contradiz com a natureza capacitada do problema real. Porém, havendo, por exemplo, duas escolas, uma com excesso e outra com escassez, pode-se resolver o problema transferindo capacidade da primeira para a segunda;

- Basicamente, a grande maioria das escolas pode variar sua capacidade através de medidas como: aumentar/diminuir o número de alunos por classe; variar o número de turnos de cada escola; ativar espaços não utilizados; ampliação; construção/fechamento de novas escolas; transferência de mesas, cadeiras, professores, funcionários etc.

Pode-se encontrar o relatório completo para o caso com restrição de capacidade (Modelo Capacitado) em Barcelos (2002). De acordo com o relatório, pode-se concluir que:

- 51 escolas são suficientes em Vitória caso a política de mandar toda a população 7-14 anos para a escola pública queira ser implantada. O que necessita ser feito é o remanejamento e a ampliação de escolas e recursos; 
- Pode-se concluir em relação às distâncias máximas percorridas que há somente um arco com distância superior a 1500 metros, distância máxima almejada. De acordo com o relatório, essa distância refere-se ao arco entre os vértices 171-201 e equivale a 2.608,84 metros. Observando a Figura 5, pode-se concluir que este problema é facilmente resolvido aumentando-se a capacidade da escola localizada no vértice 199, setor censitário 910065. Dessa forma, a distância seria de 526 metros.

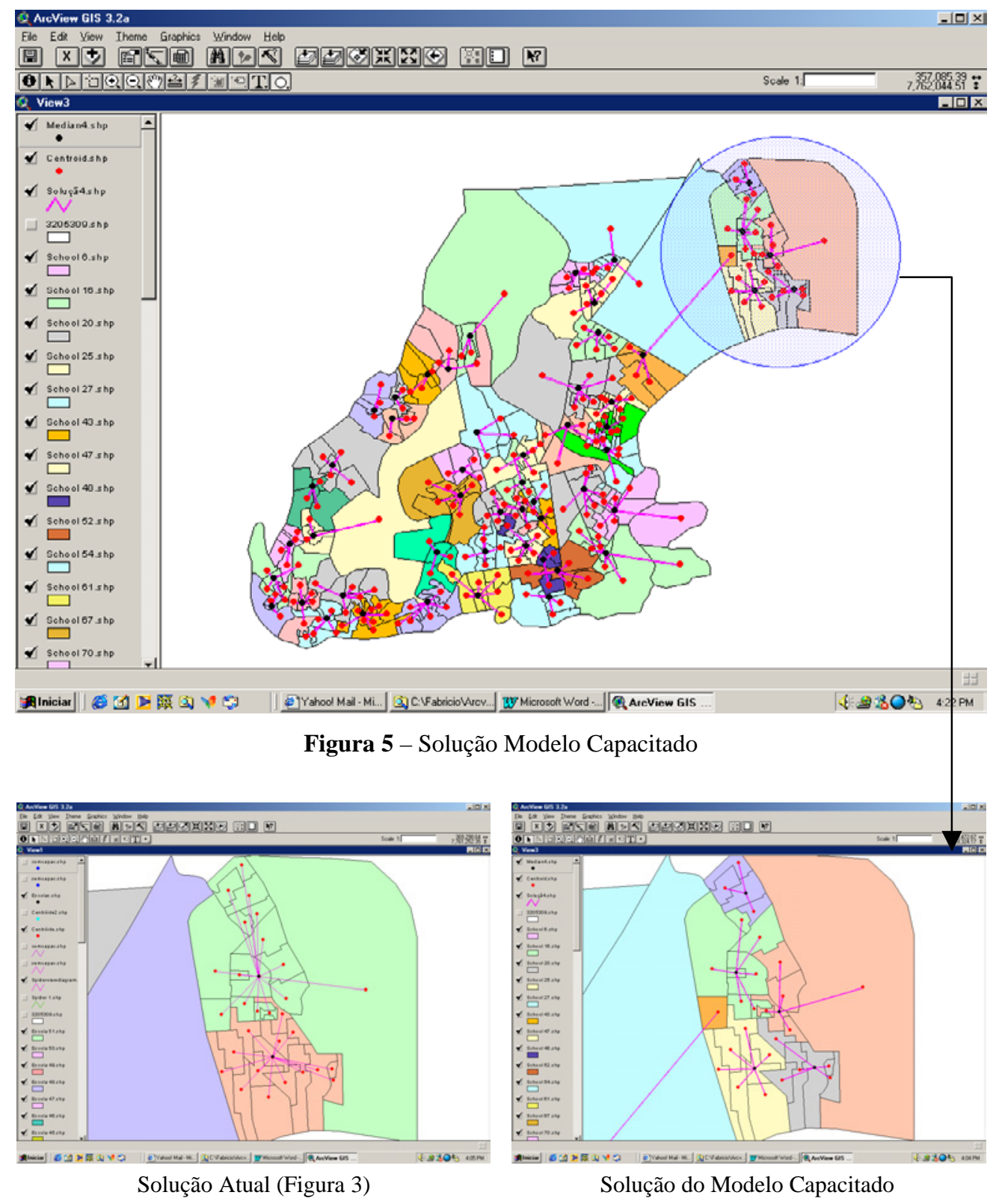

Figura 6 - Mais Escolas em Algumas Regiões 
A Tabela 1 mostra valores relativos dos resultados obtidos para os três estudos aqui tratados. Para construir a Tabela foi adotado um peso em cada vértice de 1 e as distâncias entre vértices em metros. Assim, a terceira coluna da tabela mostra o valor $\mathrm{Z}$ dado em metros até a escola mais próxima segundo as três situações: distribuição atual; localização proposta e modelo capacitado. Pode-se observar que a solução heurística do modelo da p-mediana fornece um ganho de 28.643 metros em relação a atual distribuição das escolas (99.229 70586 metros). Já em relação ao Modelo Capacitado, como era de se esperar, o ganho foi menor: 14.146 metros (99.229 - 85.083 metros).

Tabela 1 - Resultado Final

\begin{tabular}{|c|c|c|}
\hline Método Utilizado & Peso Populacional & $\begin{array}{c}\text { "Z"- Valor da Função } \\
\text { Objetivo (m) }\end{array}$ \\
\hline $\begin{array}{c}\text { Avaliação Atual } \\
\text { Arcview (SIG) }\end{array}$ & 1 habitante por setor censitário & 99.229 \\
\hline $\begin{array}{c}\text { Solução Heurística: } \\
\text { Modelo não-capacitado }\end{array}$ & 1 habitante por setor censitário & 70.586 \\
\hline $\begin{array}{c}\text { Relaxação LagSur } \\
\text { Modelo Capacitado }\end{array}$ & 1 habitante por setor censitário & 85.083 \\
\hline
\end{tabular}

Não foi levado em consideração neste estudo uma pequena quantidade de alunos que estão avançados em relação aos demais, começando o ensino fundamental com 5 ou 6 anos e terminando com 12 ou 13 anos. Há também, porém em maiores proporções, aqueles que entram atrasados ou que se atrasaram por repetições e abandonos devido à ineficiência do sistema. Muitos ainda não chegam a terminar a primeira série e outros nem sequer entram no sistema, condenados ao analfabetismo.

Para piorar o resultado acima exposto, deve-se levar em consideração que a área metropolitana da Grande Vitória é constituída por cinco municípios demográfica, econômica e culturalmente diferenciados. Desses, Vitória, por ser a capital, é a que parece estar atualmente em melhor situação, enquanto os demais se apresentam ainda em crescente desenvolvimento. Além disso, muitas famílias que têm suas residências nos municípios vizinhos trabalham na capital, e por algum tipo de conveniência ou pela melhor qualidade da escola da capital, preferem ter seus filhos estudando na capital.

Como consequiência da vinda de alguns estudantes dos municípios vizinhos para a capital, tem-se o surgimento de uma "demanda extra" sobre o sistema, que não foi levada em consideração nesse estudo.

\section{Conclusões}

O presente estudo descreve um conjunto de metodologias para estudar a localização de escolas públicas primárias e sua concomitante aplicação ao município de Vitória/ES. O modelo básico empregado foi a p-mediana, que pressupõe que o aluno prefere a escola mais próxima de sua residência, fato amplamente aceito e comprovado, pelo menos para a clientela da escola pública.

O estudo prático teve o apoio de recursos que associam banco de dados a informações espaciais, o Arcview-GIS. Dados básicos foram obtidos no IBGE, incluindo mapas e setores 
censitários digitalizados, e dados de população por setor censitário. Dados escolares foram obtidos nas secretarias municipal e estadual. O software Arcview facilita a localização de escolas e centróides, a determinação de distâncias e ajuda a representação pictórica das informações, resultados e propostas. Para o estudo de relocalização, scripts foram preparados para passar os dados para códigos escritos em $\mathrm{C}$ e retornar os resultados para visualização. A matriz de distâncias foi calculada externamente, pois o compilador $\mathrm{C}$ é mais eficiente do que a linguagem nativa do GIS.

A presente pesquisa teve três objetivos complementares, mas bastante independentes entre si. O primeiro foi a avaliação da atual localização de escolas, de modo a identificar áreas com escassez e áreas com excesso de vagas. Para efeitos práticos, essa fase é a mais útil, pois os desequilíbrios podem ser mostrados em um mapa, juntamente com suas magnitudes. Dependendo dos valores numéricos desses desequilíbrios, medidas corretivas podem variar desde o gerenciamento da capacidade até novas construções. Por gerenciamento da capacidade entende-se medidas capazes de ajustar capacidades das escolas. Para aumentar capacidade, por exemplo, pode-se propor expansão de edifícios, adaptação de espaços cobertos para salas de aula, turnos adicionais e mais estudantes por sala. A redução de capacidade é mais simples, bastando a transferência de professores, móveis escolares e apoio de secretaria para áreas mais necessitadas.

O segundo objetivo é produzir uma nova proposta de localização de toda a rede. Essa parte do estudo é mais importante quando os desequilíbrios são dramáticos ou quando várias novas escolas devem ser construídas. Para uso prático, as localizações propostas devem ser conciliadas com as escolas existentes. A idéia é que as localizações propostas definam um zoneamento escolar. Nesses zoneamentos, algumas escolas podem já existir, de modo que os desequilíbrios sejam revistos e as ampliações necessárias sejam definidas.

O terceiro objetivo é a solução capacitada. O senso comum diz que uma escola é uma instalação capacitada, embora, dentro de certos limites, esta capacidade possa ser alterada. Em razão de diversos estudos econômicos e pedagógicos, há uma tendência para se trabalhar com escolas padronizadas, evitando as muito grandes e as muito pequenas.

A receptividade das autoridades educacionais do município de Vitória ao estudo foi colocado em um documento oficial. Em síntese, os pontos básicos apontados foram: i) trata-se de um estudo técnico que pode ser usado para ratificar os estudos rotineiros sobre planejamento de expansão da rede; ii) o documento reconhece a importância das sugestões apresentadas quanto aos ajustes de capacidade e modelo capacitado; iii) os dados coletados pelo estudo contribuem para a ampliação e aperfeiçoamento do banco de dados existente; iv) o estudo virá estimular iniciativas com outros setores da administração, como os programas de desenvolvimento social, para integrar serviços oferecidos à comunidade; e v) solicitação que este estudo seja renovado a cada 5 anos, em lugar dos dez sugeridos.

\section{Agradecimentos}

O primeiro autor agradece ao Conselho Nacional de Desenvolvimento Científico e Tecnológico - CNPq; o segundo autor agradece ao Conselho Nacional de Desenvolvimento Científico e Tecnológico - CNPq e à Fundação para o Amparo a Pesquisa no Estado do Rio de Janeiro - FAPERJ; o terceiro autor agradece ao Conselho Nacional de Desenvolvimento Científico e Tecnológico - CNPq e à Fundação para o Amparo a Pesquisa no Estado de São Paulo - FAPESP - por apoio financeiro parcial. 


\section{Referências Bibliográficas}

(1) Barcelos, F.B. (2002). Avaliação da Localização de Escolas com Modelo Capacitado e Não-Capacitado e Uso de uma Ferramenta GIS: Estudo de Caso da Cidade de Vitória/ES. Dissertação M. Sc. - Programa de Engenharia de Produção, PUC/RIO, Brasil.

(2) ESRI - Environmental Systems Research Institute (1996). Avenue customization and application development for Arcview. Environmental Systems Research Institute, Inc., Redlands, CA.

(3) Galvão, R.D. \& Raggi, L.A. (1989). A Method for Solving to Optimality Uncapacitated Location Problems. Annals of Operations Research, 18, 225-244.

(4) Held, M. \& Karp, R.M. (1971). The traveling-salesman problem and minimum spanning trees: part II. Mathematical Programming, 1, 6-25.

(5) Lorena, L.A.N.; Senne, E.L.F.; Paiva, J.A.C. \& Pereira, M.A. (2001). Integração de Modelos de Localização a Sistemas de Informações Geográficas. Gestão e Produção, 8, 180-195.

(6) Lorena, L.A.N. \& Senne, E.L.F. (2003). Local search heuristics for capacitated p-median problems. Networks and Spatial Economics, 3, 407-419.

(7) Martello S. \& Toth, P. (1990). Knapsack problems: Algorithms and computer implementations. John Wiley \& Sons, New York.

(8) Narciso, M.G. \& Lorena, L.A.N. (1999). Lagrangean/surrogate Relaxation for Generalized Assignment Problems. European Journal of Operational Research, 114(1), 165-177.

(9) Pizzolato, N.D. \& Silva, H.B.F. (1993). Proposta Metodológica de Localização de Escolas: Estudo do Caso de Nova Iguaçu. Pesquisa Operacional, 14, 1-13.

(10) Pizzolato, N.D. (1994). A Heuristic for Large-Size p-Median Location Problems with Application to School Location. Annals of Operations Research, 50, 473-485.

(11) Senne, E.L.F. \& Lorena, L.A.N. (2000). Lagrangean/Surrogate Heuristics for p-Median Problems. In: Computing Tools for Modeling, Optimization and Simulation: Interfaces in Computer Science and Operations Research [edited by M. Laguna and J.L. Gonzalez-Velarde], Kluwer Academic Publishers, 115-130.

(12) SPRING (1998). Sistema de processamento de informações georeferenciadas. INPE, São José dos Campos, SP. Último acesso ao site em 23 de Janeiro de 2002. Disponível na Internet: 〈http://www.dpi.inpe.br/spring>. 